Supporting information

\title{
Substitution of Hydroxyl Groups with Alkynyl Moieties Using Alkynylboron Dihalides: Efficient Approach to Secondary Alkylacetylene Derivatives
}

George W. Kabalka, ${ }^{*}$ Min-Liang Yao and Scott Borella

The University of Tennessee Departments of Chemistry and Radiology Knoxville, TN 37996-1600 USA

\author{
kabalka@utk.edu
}




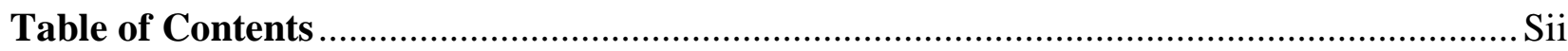

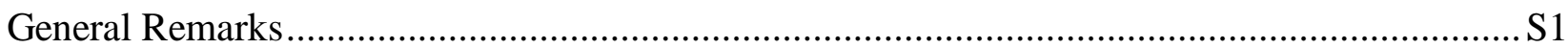

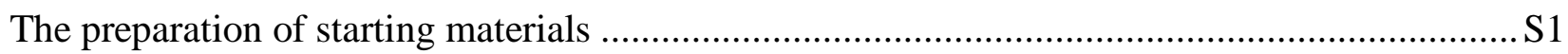

Typical experimental procedure for preparation compounds $\mathbf{3 a}-\mathbf{3 r} \ldots \ldots \ldots \ldots \ldots \ldots \ldots \ldots \ldots \ldots \ldots \ldots \ldots \ldots . . . \mathrm{S} 1-\mathrm{S} 5$

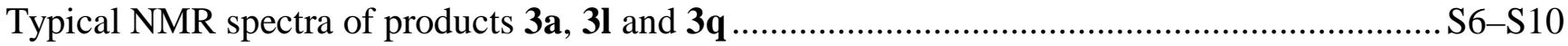

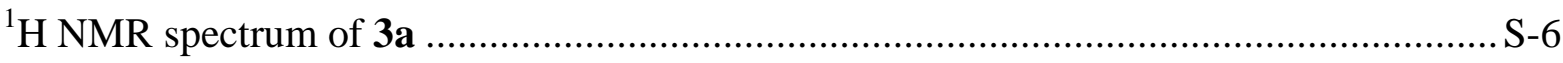

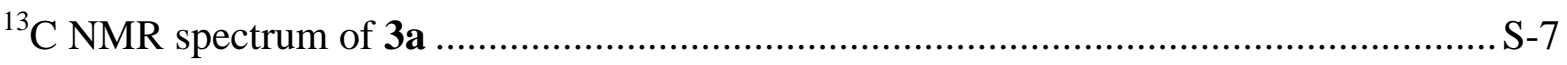

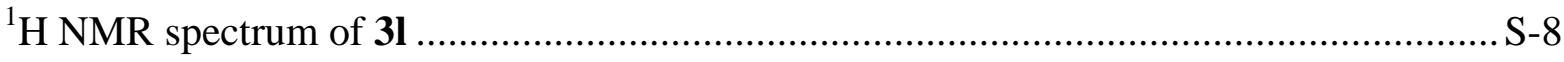

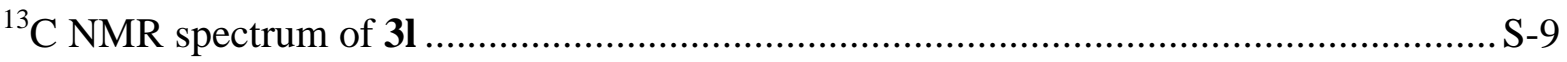

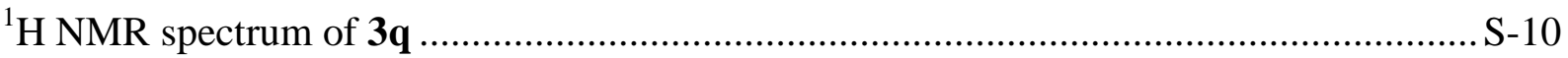

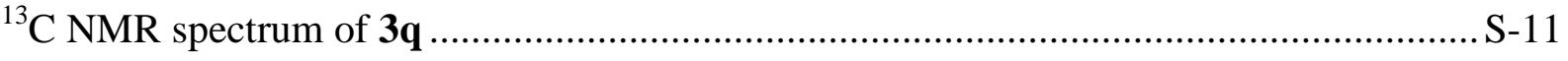

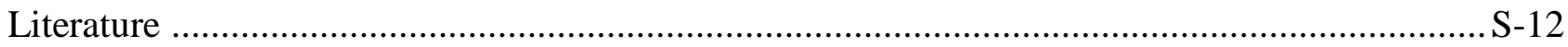


General Remarks: All reagents were used as received. Column chromatography was performed using silica gel (60 ̊, 230-400 mesh, ICN Biomedicals GmbH, Eschwege, Germany). Analytical thin-layer chromatography was performed using $250 \mu \mathrm{m}$ silica (Analtech, Inc., Newark, DE). ${ }^{1} \mathrm{H}$ NMR and ${ }^{13} \mathrm{C}$ NMR spectra were recorded at 250.13 and $62.89 \mathrm{MHz}$, respectively. Chemical shifts for ${ }^{1} \mathrm{H}$ NMR and ${ }^{13} \mathrm{C}$ NMR spectra were referenced to TMS and measured with respect to the residual protons in the deuterated solvents. Microanalyses was performed by Atlantic Microlab, Inc. Norcross, Georgia.

The preparation of starting materials: Benzylic alcohols $\mathbf{2 a}$ and $\mathbf{2 b}$ were purchased from Aldrich Chemical Company. Compounds $2 \mathbf{c}, \mathbf{2 f}, \mathbf{2 g}$ and $\mathbf{2 h}$ were obtained by reduction of the corresponding ketones according to the literature procedure. ${ }^{1}$ Compounds $\mathbf{2 d}$ and $\mathbf{2 e}$ were prepared from aryl aldehydes by reaction with phenylmagnesium bromide. ${ }^{2}$ Compounds $\mathbf{2 i}$ and $2 \mathbf{j}$ were prepared according to the literature procedure. ${ }^{3}$

Typical experimental procedure for the preparation of 3a-3r: A solution of alkyne (1.5 mmol) in dry hexane $(8 \mathrm{~mL})$ was treated with $n$-butyllithium $(1.0 \mathrm{~mL}$ of a $1.6 \mathrm{M}$ solution in hexane) at $0{ }^{\circ} \mathrm{C}$. After stirring at room temperature for $30 \mathrm{~min}$, boron trihalide $(1.5 \mathrm{mmol})$ was added to the reaction mixture. In a separate flask, the benzylic alcohol $(1.6 \mathrm{mmol})$ in dry dichloromethane $(8 \mathrm{~mL})$ was treated with $n$-butyllithium ( $1.0 \mathrm{~mL}$ of a $1.6 M$ solution in hexane) at $0{ }^{\circ} \mathrm{C}$ and then warmed to room temperature. After stirring at room temperature for $30 \mathrm{~min}$, this solution was transferred to the first flask and the mixture was allowed to stir overnight. Water $(20 \mathrm{~mL})$ was added to quench the reaction. The mixture was extracted with 
ethyl acetate dried over anhydrous $\mathrm{MgSO}_{4}$, the solvent removed in vacuo, and the product purified by silica gel column chromatography using hexane as an eluent.

1,3,3-Triphenyl-1-propyne, $\mathbf{3 a}^{4}:{ }^{1} \mathrm{H}$ NMR $\left(250 \mathrm{MHz}, \mathrm{CDCl}_{3}\right): \delta 7.19-7.47(\mathrm{~m}, 15 \mathrm{H}), 5.19(\mathrm{~s}$, 1H). ${ }^{13} \mathrm{C} \mathrm{NMR}\left(\mathrm{CDCl}_{3}\right): \delta 141.7,131.7,128.6,128.2,127.9,126.9,123.5,90.2,84.9,43.7$.

1-(4-Methylphenyl)-3,3-diphenyl-1-propyne, 3b: ${ }^{1} \mathrm{H}$ NMR $\left(250 \mathrm{MHz}, \mathrm{CDCl}_{3}\right)$ : 7.07-7.45 (m, 14H), 5.19 (s, $1 \mathrm{H}), 2.32(\mathrm{~s}, 3 \mathrm{H}) .{ }^{13} \mathrm{C}$ NMR $\left(\mathrm{CDCl}_{3}\right): \delta 142.2,141.9,138.0,131.5,128.9,128.6$, 128.4, 128.0, 127.2, 126.8, 89.4, 80.0, 43.7, 21.3. Anal. Calcd for $\mathrm{C}_{22} \mathrm{H}_{18}: \mathrm{C}, 93.57 ; \mathrm{H}, 6.43$. Found: C, 93.34; H, 6.61.

1-(2-Fluorophenyl)-3,3-diphenyl-1-propyne, 3c: ${ }^{1} \mathrm{H}$ NMR $\left(250 \mathrm{MHz}, \mathrm{CDCl}_{3}\right): \delta 7.14-7.42$ (m, 13H), 6.96-6.99 (m, 1H), 5.19 (s, 1H). ${ }^{13} \mathrm{C}$ NMR $\left(\mathrm{CDCl}_{3}\right): \delta 164.3,160.4,141.4,129.7$, 128.7, 127.9, 127.6, 127.0, 125.4, 118.6, 118.3, 115.5, 115.2, 91.3, 83.7, 43.7. Anal. Calcd for $\mathrm{C}_{21} \mathrm{H}_{15} \mathrm{~F}: \mathrm{C}, 88.09 ; \mathrm{H}, 5.28$. Found: C, 87.57; H, 5.09.

1-Phenyl-3,3-di(4-methoxyphenyl)-1-propyne, 3d: ${ }^{1} \mathrm{H}$ NMR (250 MHz, $\left.\mathrm{CDCl}_{3}\right): \delta 7.44-7.48$ (m, 2H), 7.26-7.34 (m, 7H), 6.83-6.86 (m, 4H), 5.11(s, 1H), $3.76(\mathrm{~s}, 6 \mathrm{H}) .{ }^{13} \mathrm{C}$ NMR $\left(\mathrm{CDCl}_{3}\right)$ : $\delta$ 156.4, 134.2, 131.6, 128.8, 128.2, 127.8, 123.6, 113.9, 90.8, 84.5, 55.2, 42.0. Anal. Calcd for $\mathrm{C}_{23} \mathrm{H}_{20} \mathrm{O}_{2}: \mathrm{C}, 84.12 ; \mathrm{H}, 6.14$. Found: C, 83.97; H, 5.78.

1-(4-Methylphenyl)-3,3-di(4-methoxylphenyl)-1-propyne, 3e: ${ }^{1} \mathrm{H}$ NMR $\left(250 \mathrm{MHz}, \mathrm{CDCl}_{3}\right)$ : $\delta 6.81-7.36(\mathrm{~m}, 12 \mathrm{H}), 5.09(\mathrm{~s}, 1 \mathrm{H}), 3.73(\mathrm{~s}, 6 \mathrm{H}), 2.30(\mathrm{~s}, 3 \mathrm{H}) .{ }^{13} \mathrm{C} \mathrm{NMR}\left(\mathrm{CDCl}_{3}\right): \delta 158.3$, 134.3, 131.4, 130.6, 129.1, 128.8, 126.4, 113.9, 90.0, 84.5, 55.3, 42.0, 21.3. HR-MS Calcd for $\mathrm{C}_{24} \mathrm{H}_{22} \mathrm{O}_{2}: 342.1620$. Found: 342.1612 . 
1-Phenyl-3,3-di(4-fluorophenyl)-1-propyne, 3f: ${ }^{1} \mathrm{H}$ NMR $\left(250 \mathrm{MHz}, \mathrm{CDCl}_{3}\right): \delta 6.95-7.48$ $(\mathrm{m}, 13 \mathrm{H}), 5.14(\mathrm{~s}, 1 \mathrm{H}) .{ }^{13} \mathrm{C} \mathrm{NMR}\left(\mathrm{CDCl}_{3}\right): \delta 163.8,159.9,137.3,131.6,129.4,129.3,128.3$, 128.2, 123.1, 115.6, 115.3, 89.6, 85.3, 42.2. HR-MS Calcd for $\mathrm{C}_{21} \mathrm{H}_{14} \mathrm{~F}_{2}$ : 304.1064. Found: 304.1057.

1-(4-Methoxyphenyl)-3,3-di(4-fluorophenyl)-1-propyne, 3g: ${ }^{1} \mathrm{H} \mathrm{NMR}\left(250 \mathrm{MHz}, \mathrm{CDCl}_{3}\right): \delta$ 6.61-7.41 (m, 12H), $5.14(\mathrm{~s}, 1 \mathrm{H}), 3.80(\mathrm{~s}, 3 \mathrm{H}) .{ }^{13} \mathrm{C} \mathrm{NMR}\left(\mathrm{CDCl}_{3}\right): \delta 163.8,159.8,137.5,133.0$, $129.7,129.6,129.4,129.2,128.0,115.6,115.3,113.9,113.7,88.1,85.2,55.3,42.3$. HR-MS Calcd for $\mathrm{C}_{22} \mathrm{H}_{16} \mathrm{~F}_{2}: 334.1169$. Found: 334.1161 .

1,3-Diphenyl-3-(4-chlorophenyl)-1-propyne, 3h: ${ }^{1} \mathrm{H}$ NMR $\left(250 \mathrm{MHz}, \mathrm{CDCl}_{3}\right): \delta 7.21-7.47$ $(\mathrm{m}, 14 \mathrm{H}), 5.14(\mathrm{~s}, 1 \mathrm{H}) .{ }^{13} \mathrm{C} \mathrm{NMR}\left(\mathrm{CDCl}_{3}\right): \delta 141.2,140.3,132.7,131.6,129.2,128.7,128.2$, 128.1, 127.8, 127.1, 123.2, 89.6, 85.2, 43.1. Anal. Calcd for $\mathrm{C}_{21} \mathrm{H}_{15} \mathrm{Cl}: \mathrm{C}, 83.30 ; \mathrm{H}, 4.99$. Found: C, 83.77; H, 5.04.

1-(2-Fluorophenyl)-3-phenyl-3-(4-chlorophenyl)-1-propyne, 3i: ${ }^{1} \mathrm{H} \quad \mathrm{NMR} \quad(250 \mathrm{MHz}$, $\left.\mathrm{CDCl}_{3}\right): \delta 6.95-7.39(\mathrm{~m}, 13 \mathrm{H}), 5.15(\mathrm{~s}, 1 \mathrm{H}) .{ }^{13} \mathrm{C} \mathrm{NMR}\left(\mathrm{CDCl}_{3}\right): \delta 164.3,160.4,140.7,139.9$, $132.9,129.9,129.7,129.2,128.8,127.8,127.5,127.2,118.7,118.3,115.6,115.3,90.7,84.1$, 43.1. Anal. Calcd for $\mathrm{C}_{21} \mathrm{H}_{14} \mathrm{ClF}$ : C, 78.63; H, 4.40. Found: C, 78.08; H, 4.32.

1,3-Diphenyl-3-(2-methylphenyl)-1-propyne, 3j: ${ }^{1} \mathrm{H}$ NMR $\left(250 \mathrm{MHz}, \mathrm{CDCl}_{3}\right): \delta 7.15-7.52$ $(\mathrm{m}, 14 \mathrm{H}), 5.37(\mathrm{~s}, 1 \mathrm{H}), 2.31(\mathrm{~s}, 3 \mathrm{H}) .{ }^{13} \mathrm{C} \mathrm{NMR}\left(\mathrm{CDCl}_{3}\right): \delta 140.7,139.4,135.9,131.6,130.7$, $128.8,128.5,128.2,128.0,127.9,127.5,127.3,127.1,126.7,126.3,123.6,90.2,84.5,40.8$, 19.7. Anal. Calcd for $\mathrm{C}_{22} \mathrm{H}_{18}$ : C, 93.57; H, 6.43. Found: C, 93.13; H, 6.29.

1-(2-Fluorophenyl)-3-phenyl-1-butyne, 3k: ${ }^{1} \mathrm{H}$ NMR $\left(250 \mathrm{MHz}, \mathrm{CDCl}_{3}\right): \delta 7.21-7.46(\mathrm{~m}$, $8 \mathrm{H}), 6.95-7.01(\mathrm{~m}, 1 \mathrm{H}), 3.97(\mathrm{q}, J=7.15 \mathrm{~Hz}, 1 \mathrm{H}), 1.57(\mathrm{~d}, J=7.15,3 \mathrm{H}) .{ }^{13} \mathrm{C} \mathrm{NMR}\left(\mathrm{CDCl}_{3}\right): \delta$ $164.3, \mathrm{~S}-3$ 
$160.4,143.0,129.8,129.6,128.6,128.6,128.2,127.5,126.9,126.8,126.5,118.6,118.3,115.2$, 114.9, 93.7, 81.3, 32.4, 24.3. HR-MS Calcd for $\mathrm{C}_{16} \mathrm{H}_{13} \mathrm{~F}: 224.1001$. Found: 224.0995 .

(E)-1,3,5-Triphenylpent-4-yne-1-ene, 31: ${ }^{1} \mathrm{H}$ NMR (250 MHz, $\left.\mathrm{CDCl}_{3}\right): \delta 7.16-7.48(\mathrm{~m}, 15 \mathrm{H})$, $6.75(\mathrm{~d}, J=15.6 \mathrm{~Hz}, 1 \mathrm{H}), 6.30(\mathrm{dd}, J=15.6 ; 6.54 \mathrm{~Hz}, 1 \mathrm{H}), 4.71(\mathrm{~d}, J=6.54 \mathrm{~Hz}, 1 \mathrm{H}) .{ }^{13} \mathrm{C}$ $\operatorname{NMR}\left(\mathrm{CDCl}_{3}\right): \delta 140.2,136.7,131.6,130.4,129.5,128.6,128.5,128.2,127.7,127.0,126.5$, 123.4, 88.8, 85.4, 41.2. Anal. Calcd for $\mathrm{C}_{23} \mathrm{H}_{18}$ : C, 93.84; H, 6.16. Found: C, 93.52; H, 6.08.

(E)-5-(2-Fluorophenyl)-1,3-diphenylpent-4-yne-1-ene, 3m: ${ }^{1} \mathrm{H} \mathrm{NMR}\left(250 \mathrm{MHz}, \mathrm{CDCl}_{3}\right): \delta$ 7.19-7.47 (m, 13H), 6.89-6.97 (m, 1H), $6.71(\mathrm{~d}, J=15.5 \mathrm{~Hz}, 1 \mathrm{H}), 6.30(\mathrm{dd}, J=15.4 ; 6.19 \mathrm{~Hz}$, $1 \mathrm{H}), 4.71(\mathrm{~d}, J=6.19 \mathrm{~Hz}, 1 \mathrm{H}) .{ }^{13} \mathrm{C} \mathrm{NMR}\left(\mathrm{CDCl}_{3}\right): \delta 164.3,160.4,141.8,139.9,136.7,130.6$, $129.8,129.2,128.5,127.6,126.5,125.3,125.2,118.7,118.3,115.5,115.0,89.9,84.2,41.1$. Anal. Calcd for $\mathrm{C}_{23} \mathrm{H}_{17} \mathrm{~F}$ : C, 88.43; H, 5.49. Found: C, 88.65; H, 5.81.

(E,E)-1,5.7-Triphenylhepta-1,3-dien-6-yne, 3n: ${ }^{1} \mathrm{H}$ NMR (250 MHz, $\left.\mathrm{CDCl}_{3}\right): \delta 7.20-7.51$ (m, $15 \mathrm{H}), 6.74-6.80(\mathrm{~m}, 1 \mathrm{H}), 6.54-6.60(\mathrm{~m}, 1 \mathrm{H}), 6.22-6.26(\mathrm{~m}, 1 \mathrm{H}), 5.95(\mathrm{dd}, J=15.6 ; 6.51 \mathrm{~Hz}$, $1 \mathrm{H}), 4.68(\mathrm{~d}, J=6.51 \mathrm{~Hz}, 1 \mathrm{H}) .{ }^{13} \mathrm{C} \mathrm{NMR}\left(\mathrm{CDCl}_{3}\right): \delta 140.3,137.3,133.6,131.7,130.8,128.6$, 127.7, 126.3, 88.8, 85.7, 41.9. Anal. Calcd for $\mathrm{C}_{25} \mathrm{H}_{20}$ : C, 93.71; H, 6.29. Found: C, 93.44; $\mathrm{H}$, 5.94.

1,5-Diphenyl-3-(4-chlorophenyl)-1,4-pentadiyne, 3o: ${ }^{1} \mathrm{H}$ NMR $\left(250 \mathrm{MHz}, \mathrm{CDCl}_{3}\right): \delta 7.26-$ $7.61(\mathrm{~m}, 14 \mathrm{H}), 5.16(\mathrm{~s}, 1 \mathrm{H}) .{ }^{13} \mathrm{C} \mathrm{NMR}\left(\mathrm{CDCl}_{3}\right): \delta 136.5,133.4,131.8,128.8,128.7,128.4$, 128.2, 122.7, 86.0, 83.1, 29.5. HR-MS Calcd for $\mathrm{C}_{23} \mathrm{H}_{15} \mathrm{Cl}$ : 326.0862. Found: 326.0854 .

1,5-Diphenyl-3-(4-methylphenyl)-1,4-pentadiyne, 3p: ${ }^{1} \mathrm{H} \mathrm{NMR}\left(250 \mathrm{MHz}, \mathrm{CDCl}_{3}\right): \delta 7.16-$ $7.56(\mathrm{~m}, 14 \mathrm{H}), 5.15(\mathrm{~s}, 1 \mathrm{H}), 2.33(\mathrm{~s}, 3 \mathrm{H}) .{ }^{13} \mathrm{C} \mathrm{NMR}\left(\mathrm{CDCl}_{3}\right): \delta 137.1,135.0,131.7,129.4$, 128.1, 127.2, 123.0, 86.8, 82.6, 29.7, 21.1. Anal. Calcd for $\mathrm{C}_{24} \mathrm{H}_{18}$ : C, 94.08; H, 5.92. Found: C, 94.47; H, 5.84 . 
1-Phenyl-3,5-di(4-methylphenyl)-1,4-pentadiyne, 3q: ${ }^{1} \mathrm{H} \mathrm{NMR}\left(250 \mathrm{MHz}, \mathrm{CDCl}_{3}\right): \delta 7.07-$ $7.56(\mathrm{~m}, 13 \mathrm{H}), 5.15(\mathrm{~s}, 1 \mathrm{H}), 2.35(\mathrm{~s}, 3 \mathrm{H}), 2.31(\mathrm{~s}, 3 \mathrm{H}) .{ }^{13} \mathrm{C} \mathrm{NMR}\left(\mathrm{CDCl}_{3}\right): \delta 138.2,137.1,135.2$, 131.7, 129.0, 128.2, 127.2, 123.1, 119.9, 86.9, 86.0, 82.7, 82.5, 29.7, 21.2. HR-MS Calcd for $\mathrm{C}_{25} \mathrm{H}_{20}$ : 320.1565 . Found: 320.1558 .

1-Phenyl-3-(4-methylphenyl)trideca-1,4-diyne, 3r: ${ }^{1} \mathrm{H}$ NMR $\left(250 \mathrm{MHz}, \mathrm{CDCl}_{3}\right): \delta 7.07-$ $7.50(\mathrm{~m}, 9 \mathrm{H}), 4.93$ (s, 1H), 2.21-2.44 (m, 5H), 1.26-1.53 (m, 12H), 0.89 (t, $J=4.25 \mathrm{~Hz}, 3 \mathrm{H})$.

${ }^{13} \mathrm{C}$ NMR $\left(\mathrm{CDCl}_{3}\right): \delta 136.9,135.7,131.8,129.3,128.6,128.5,128.1,128.0,127.1,126.6$, 126.1, 87.8, 83.2, 31.8, 31.6, 29.1, 28.9, 28.7, 22.7, 21.0, 18.9, 14.1. HR-MS Calcd for $\mathrm{C}_{27} \mathrm{H}_{32}$ : 356.2504. Found: 356.2493. 
${ }^{1 \mathrm{H}}$-NMR of compound $\mathbf{3 a}$ :

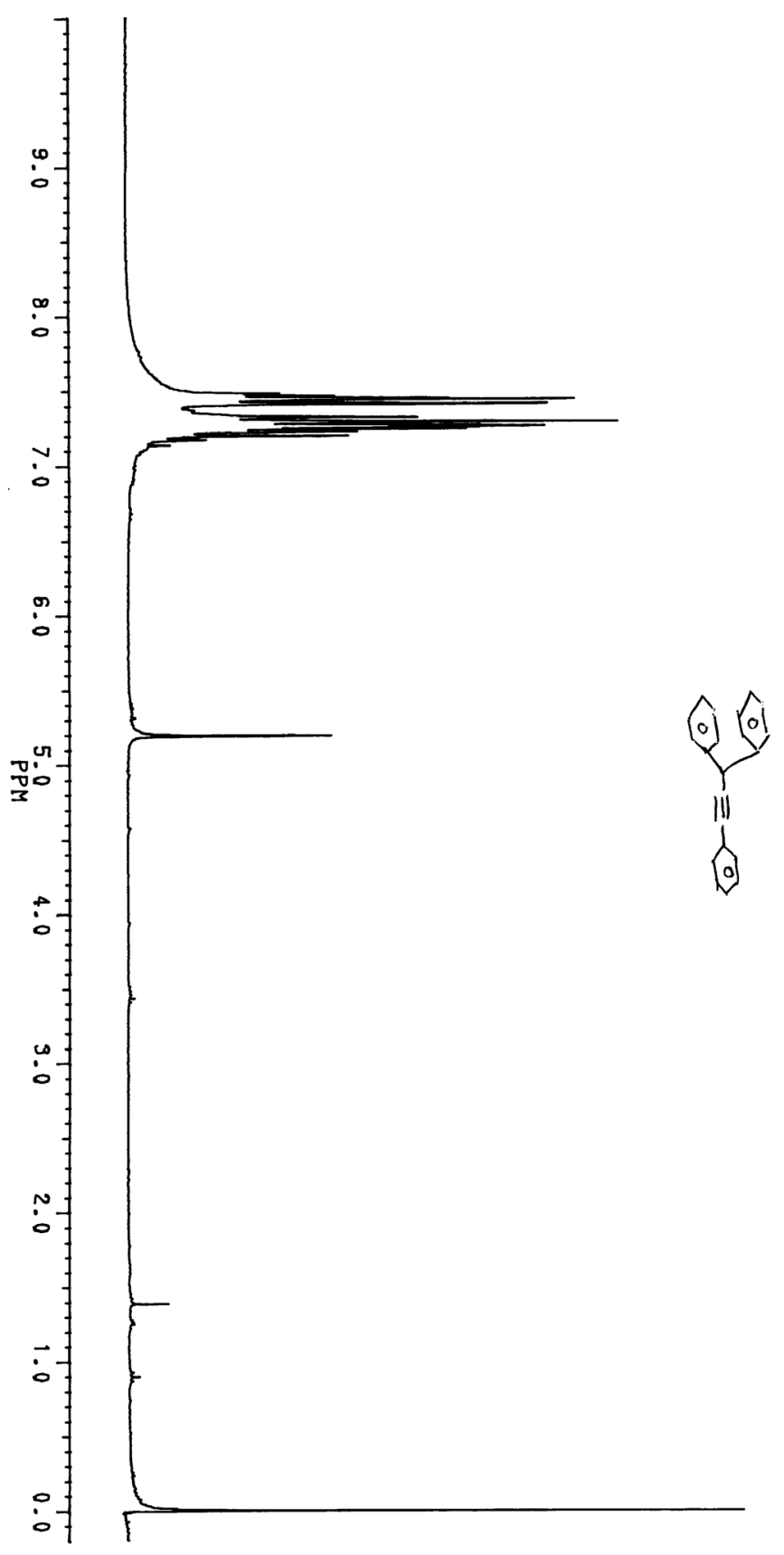

PPM
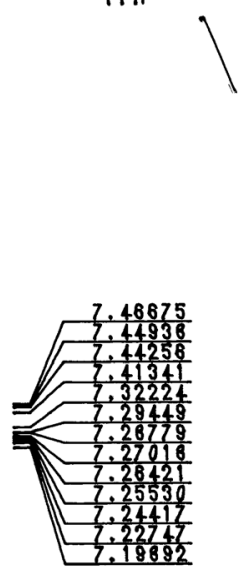

5.18204

.00013 
${ }^{13} \mathrm{C}-\mathrm{NMR}$ of compound $\mathbf{3 a}$ :

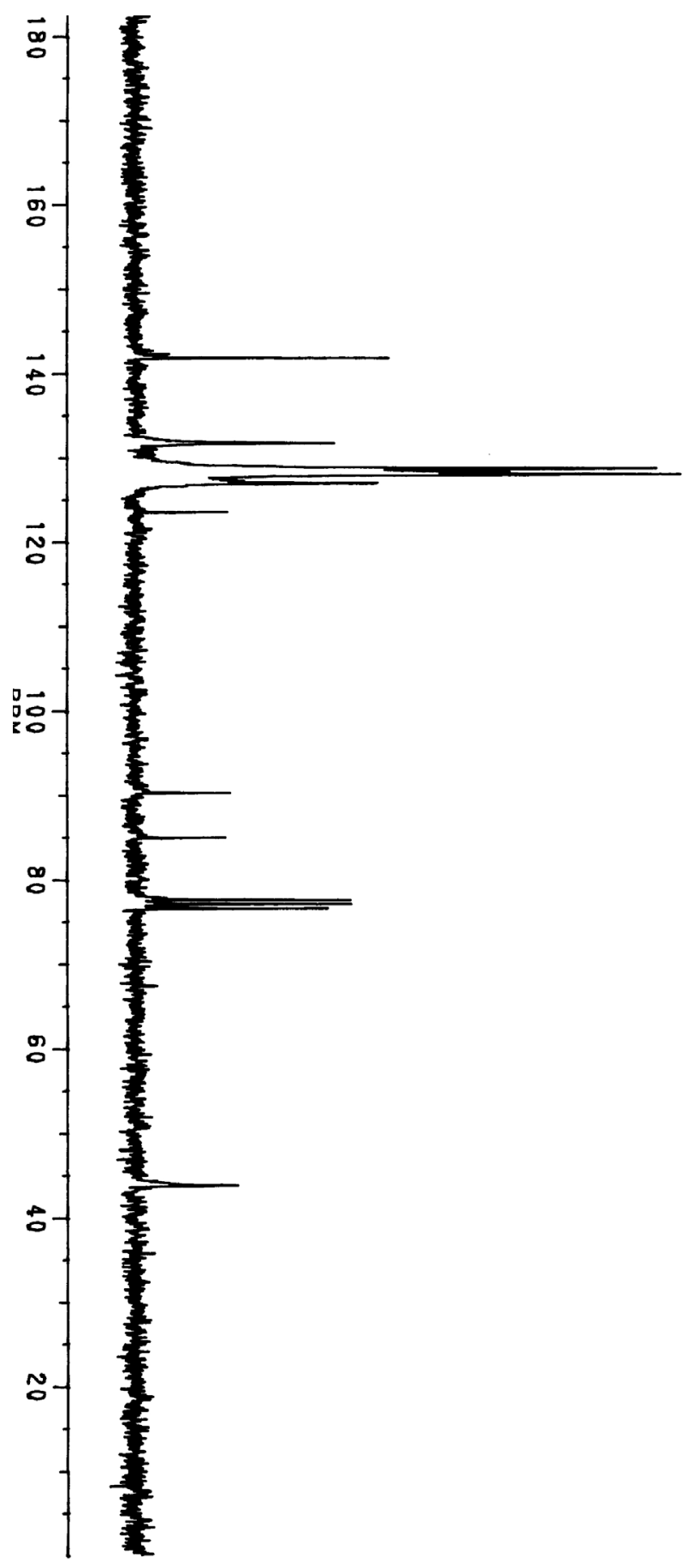


${ }^{1} \mathrm{H}-\mathrm{NMR}$ of compound 3I:

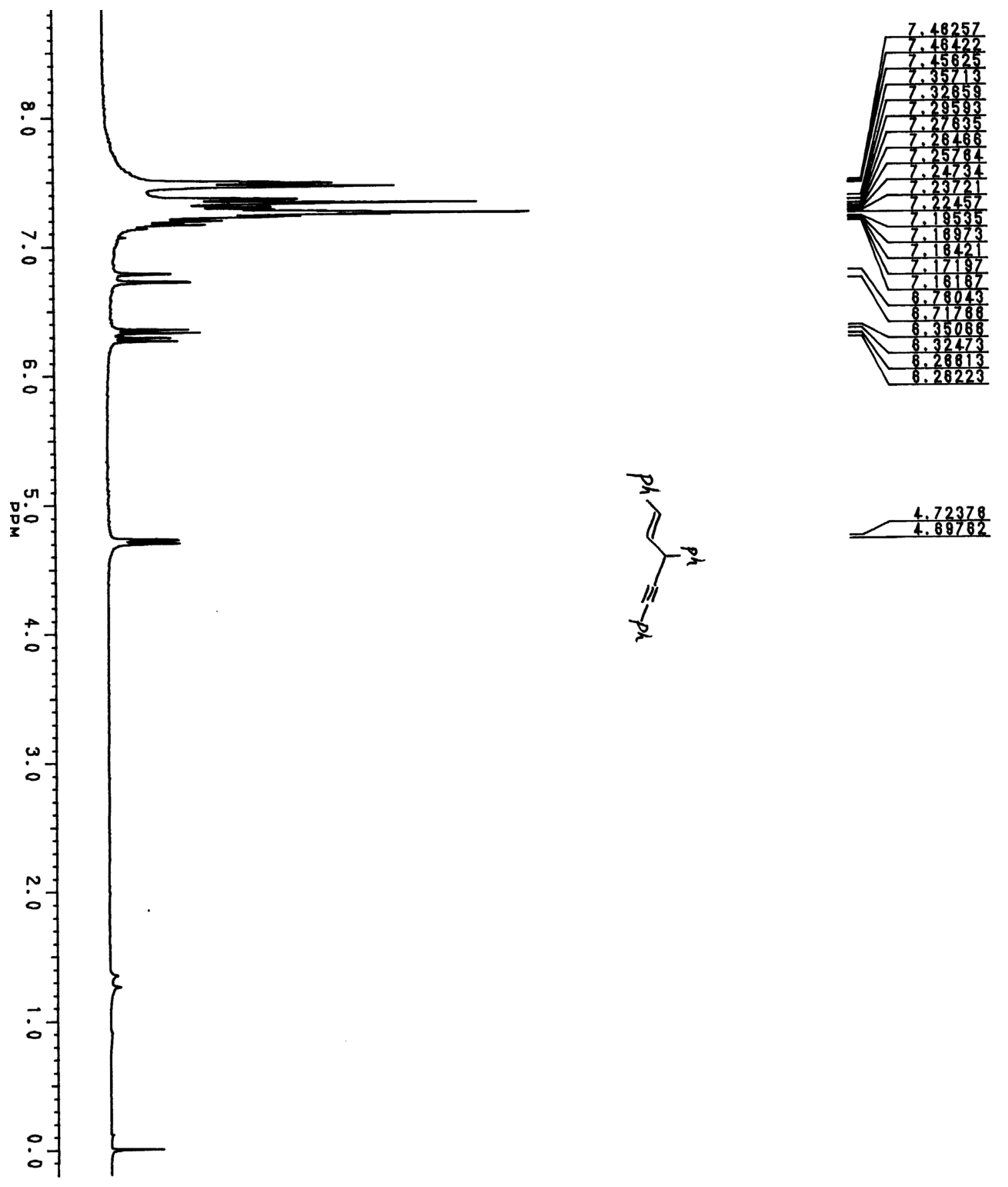


${ }^{13} \mathrm{C}-\mathrm{NMR}$ of compound $\mathbf{3 I}$ :
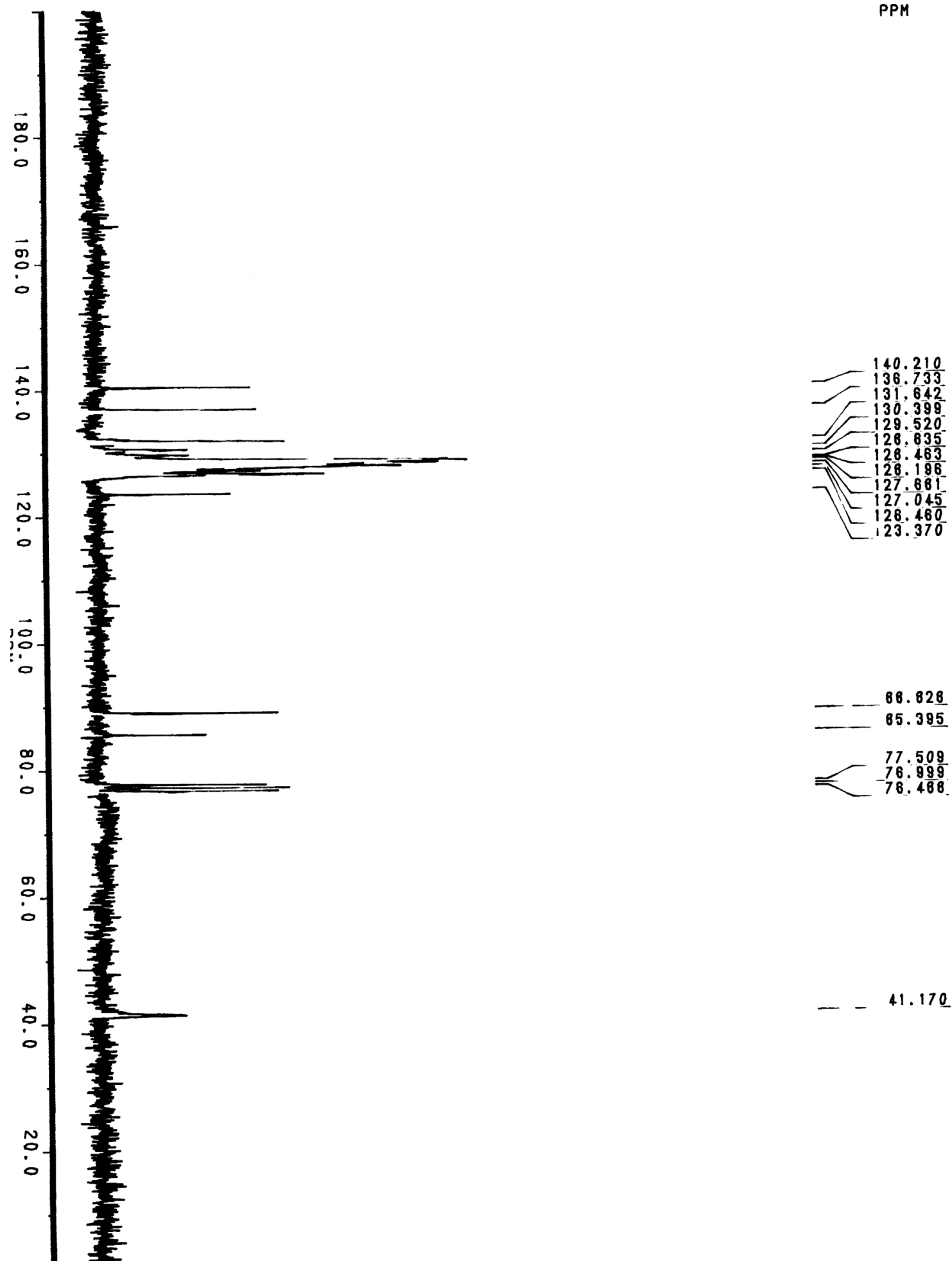

88.828

85. 395
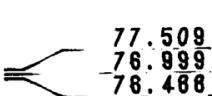

$-41.170$ 
${ }^{1} \mathrm{H}-\mathrm{NMR}$ of compound $\mathbf{3 q}$ :
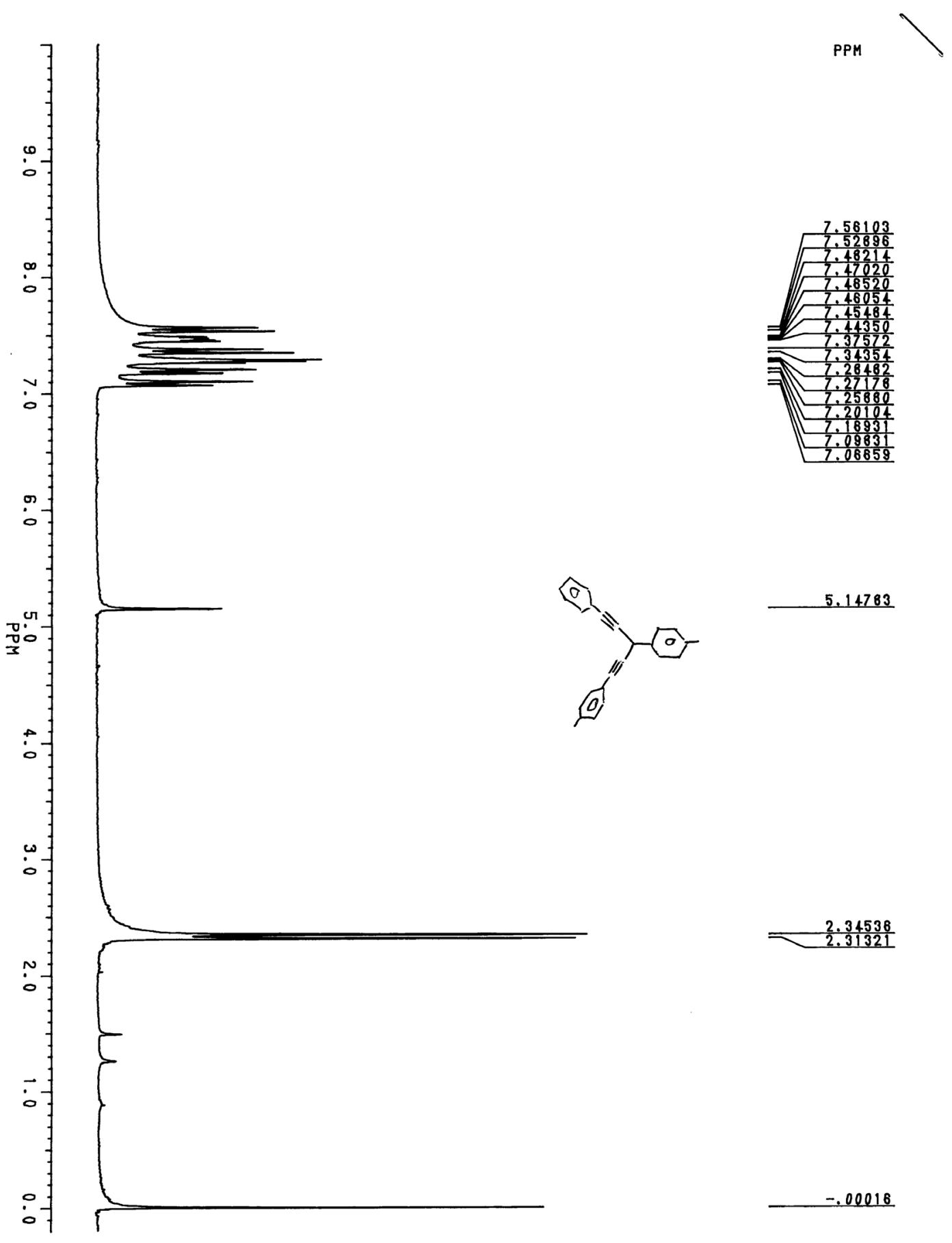

$-.00016$ 
${ }^{13} \mathrm{C}-\mathrm{NMR}$ of compound $\mathbf{3 q}$ :

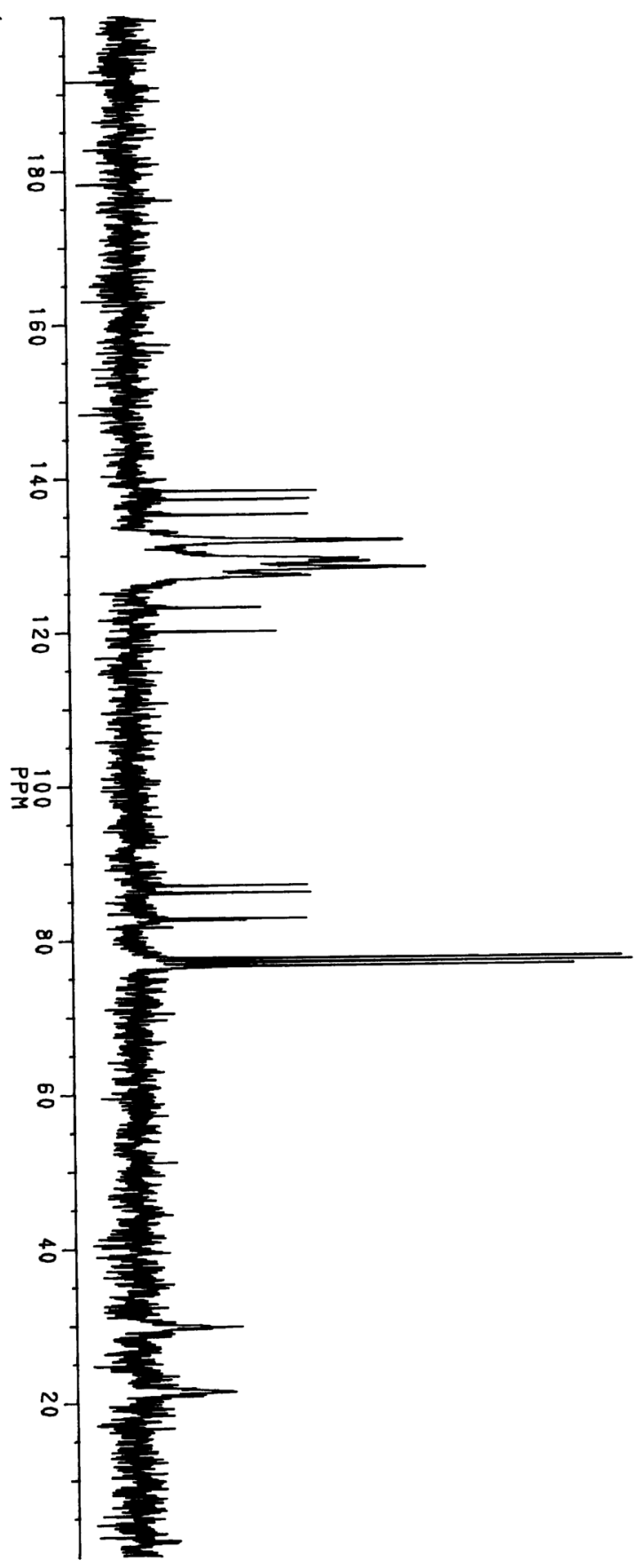

PPM
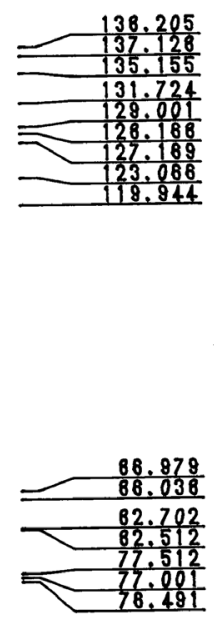

28.889

21.244 


\section{Literature:}

1. Aramini, A.; Brinchi, L.; Germani, R.; Savelli, G. Eur. J. Org. Chem. 2000, 9, 1793.

2. Makino, T.; Orfanopoulos, M.; You, T. P.; Wu, B.; Mosher, C. W.; Mosher, H. S. J. Org. Chem. 1985, 50, 5357.

3. Spee, M. P. R.; Boersma, J.; Meijer, M. D.; Slagt, M. Q.; van Koten, G.; Geus, J. W. J. Org. Chem. 2001, 66, 1647.

4. Mitsudo, T.; Kadokura, M.; Watanabe, Y. Tetrahedron Lett. 1985, 26, 3697. 